\title{
Dynamics of high-rise structures taking into account the viscoelastic properties of the material
}

\author{
Sherzod Khudainazarov ${ }^{1 *}$, Burkhon Donayev ${ }^{2}$, Talibjan Sabirjanov ${ }^{3}$, and Jahongir \\ Qosimov $^{1}$ \\ ${ }^{1}$ Department of Theoretical and Structural Mechanics, Tashkent Institute of Irrigation and \\ Agricultural Mechanization Engineers, 100000 Tashkent, Uzbekistan \\ ${ }^{2}$ Karshi Engineering-Economics Institute, 225, Mustakillik str., Karshi, Uzbekistan \\ ${ }^{3}$ Fergana Polytechnical Institute, Fergana, Uzbekistan
}

\begin{abstract}
The article deals with forced vibrations of a high-rise axisymmetric structure, represented as a viscoelastic beam of an annular section with a variable slope of the generatrices and variable thickness. The research was conducted to analyze the behavior of a high-rise structure for various kinematic effects. The task is to determine the displacements of the points of a high-rise structure at different time points under different kinematic effects. The methods were developed and a computer program was built; forced vibrations of high-rise axisymmetric structures under various kinematic actions, considering viscoelastic properties of the material, were investigated in linear, nonlinear, and viscoelastic formulations. The study of the dynamic behavior of a high-rise structure, taking into account the nonlinear and dissipative properties (different in nature) of the material, shows that the combined consideration of all these properties brings the resulting pattern closer to the one observed in reality. That is, the amplitude of the structure's oscillations does not grow infinitely, but gradually decreases over time, and the maximum possible consideration of nonlinear and dissipative properties leads to the fastest damping of oscillations.
\end{abstract}

\section{Introduction}

The problems of calculating viscoelastic structures are an integral part of the mechanics of a deformable rigid body, along with the problems of the theory of elasticity and plasticity. The variety of physical processes of deformation of real natural and artificial materials of structures (polymer composite materials, plastics, rocks, etc.) dictates the need to search for effective mathematical methods to calculate and construct the governing equations of viscoelastic bodies.

V. Volterra and L. Boltzmann conducted fundamental studies on hereditary elasticity. The mathematical theory of viscoelastic bodies was intensively developed in the 60s. Here, first,

"Corresponding author: scherzodshox77@mail.ru 
fundamental works of Russian scientists A.A. Ilyushin, B.E. Pobedrya, N.Kh. Arutyunyan, P. M. Ogibalov, V. V. Moskvitin, M. A. Koltunov, Yu. N. Rabotnov should be mentioned, as well as basic researches performed by I. I. Bugakov, V. G. Gromov, V. V. Kolokolchikov, A. S. Kravchuk, V. P. Maiboroda, L. E. Maltsev, V. P. Matveenko, M. I. Rozovsky, N.A. Trufanov, I.E. Troyanovsky [1-8].

Foreign researchers: T. Alfrey, D. Bland, R. Christensen, B. Coleman, T. Ferry, D. Fitzgerald, A. Green, B. Gross, M. Gurtin, R. Rivlin, A. Reddy, R. Sheperi and others also studied the fundamental problems of viscoelasticity [9-10].

The main methods for solving boundary value problems of equations of linear viscoelasticity are as follows: operator method, the method of integral transformations (of Laplace or Laplace-Carson), A. A. Ilyushin's approximation method, the method of direct integration of the quasi-static equilibrium equations of of equations of linear viscoelasticity of the body in time, the method of V. I. Maloy-N.A. Trufanov quasi-constant operators and others. The operator method is based on the commutative property of the operations of integration in time and differentiation in spatial coordinates. The main problem is that after replacing the constants in the elastic solution with the material operators of the of equations of linear viscoelasticity, it is necessary to decipher the resulting operator functions. It should be noted that the decoding methods used essentially depend on the type of material functions of memory. The applicability of the operator method requires the time invariability condition of the type of boundary conditions on the surface of the body.

In practical problems, a situation often occurs when an elastic solution is an irrational function of elastic constants. Methods for decoding irrational functions are proposed in the studies by Yu.N. Rabotnov, M.I. Rozovsky, Ya.V. Bykov. A number of approaches are known, based on the concept of representing an elastic solution in a form convenient for subsequent decoding using the Volterra method. In the studies by V.P. Matveenko, E.S.Ekelchik, the expansion of an elastic solution in a Taylor series by Poisson's ratio is used. The main difficulty that arises when using this approach is the need for numerical differentiation of the elastic solution.

Despite the variety of approaches used to solve problems of equations of linear viscoelasticity within the framework of the operator method, by now, it is impossible to assert that there exists a universal, accurate, economical and efficient algorithm. In particular, until recently there were relatively few publications with examples of the calculation of structures with volumetric creep and relaxation. Only in recent years, thanks to the researches of V.P. Matveenko, N.A. Trufanov, G.S. Tsaplina, the problem of accounting for the volume viscoelastic properties has become solvable. Based on these works, new patterns of changes in the stress-strain state of structures in time were revealed, in particular, the possibility of its non-monotonic change in time under monotonic external loads. Thus, in the publications of V.P. Matveenko, a method was developed for representing the elastic solution in the form of a series in powers of the A.A.Ilyushin parameter. The method is applicable both for homogeneous body problems and for piecewise homogeneous bodies. The method of quasi-constant operators was developed by V. I. Maloy and N. A. Trufanov. It can be considered as one of the most efficient operator methods. N.A. Trufanov subsequently generalized this method to problems of equations of linear viscoelasticity for an aging, anisotropic, and inhomogeneous body.

The method of integral transforms is based on the fact that the formulation of the problem of equations of linear viscoelasticity in images coincides with the formulation of the corresponding problem of the theory of elasticity; hence the coincidence of the solution in the images and the elastic solution. In the general case, the task of transitioning from images to originals is difficult. Methods based on integral transforms (Laplace or LaplaceCarson) are described in detail in various articles and monographs. The use of integral transforms is sensitive to the form in which the creep and relaxation kernels are specified; 
in particular, this method is inapplicable for problems with kernels of non-resolvent type, problems with time-variable boundary surfaces, problems that do not obey the principle of temperature-time analogy. The inverse transform is easily achieved if the elastic solution is represented as the product of the fractional-rational function of Poisson's ratio by the coordinate function. However, in the case when there is no rational dependence of the elastic solution on Poisson's ratio, one has to resort to the approximate inversion of the Laplace transform.

The approximation method was developed by A. A. Ilyushin [7-8] in order to simplify the procedure for the transition from images to originals for problems of equations of linear viscoelasticity of an ageless body with a non-relaxing volume. In subsequent works, the original version of the approximation method was generalized to the cases of more complex problems. An attempt to extend the approximation method to nonlinear viscoelastic media was made in the works of V.V. Kolokolchikov. The advantages of the approximation method are the possibility of using it in numerical solutions obtained using the FEM.

Our own analysis of the most common methods for solving problems allows us to draw the following conclusions. First, there is a limited versatility of use in the considered methods. So the method of integral transforms is focused on application to resolvent difference operators, the operator method is also in a certain dependence on the form of material functions and the method of setting boundary conditions in time. Secondly, from the point of view of practical implementation, algorithms for solving viscoelastic problems should be oriented towards adaptation to the methods of finite element and finite differences, widely used in the problems of applied analysis of strength and durability of structures, and have a variational basis. In this regard, the following formulation of the problem of constructing an algorithm may arise: to proceed not from various methods of representing an elastic solution, but from a formulation convenient for realizing a viscoelastic solution using some numerical method. In other words, since to obtain an elastic solution, from which it is quite easy to pass to a viscoelastic solution, is only an intermediate goal (the final goal is to obtain a solution to the original problem of linear viscoelastic equations), then there are ways to achieve the final goal, bypassing the stage of constructing a sufficiently "good" elastic solution and using, at the same time, the advantages of the accumulated experience in solving continual scleronomic problems of elasticity, provided by the FEM and FDM in the form of software packages and integrated systems.

In [11-15], a method is presented for determining the dynamic characteristics of a viscoelastic beam in the framework of the one-dimensional theory of viscoelasticity. The hereditary Boltzmann-Voltaire theory was used to describe dissipative processes in the building material. The natural vibrations of a viscoelastic beam are investigated and the results obtained are compared with the results of field experiments.

In [16-18], forced vibration analysis of isotropic thin circular plate resting on nonlinear viscoelastic foundation is investigated. The system coupled nonlinear partial differential equations are transformed to system of nonlinear ordinary differential equation using Galerkin decomposition method. The developed solutions are verified using the existing results in the literature, and good agreement is observed. Subsequently, the analytical solutions are used to investigate the effects of various parameters on the dynamic response of the plate. From the results, it is observed that nonlinear frequency ratio of vibrating circular plate increases with increased linear elastic foundation and tensile force.

In [19-22], vibrations of high buildings caused by wind and tornado waves were studied to assess the aeroelastic effects of high buildings using the wind tunnel tests. The aerodynamic damping coefficient and aerodynamic stiffness were determined by analyzing the aeroelastic force acting on the oscillating model. For a 347-meter-high building, the effect of aeroelastic parameters on wind-induced responses and equivalent static wind loads was 
analyzed. The results showed that during a return period of 100 years, aerodynamic damping was positive and aerodynamic stiffness was negative.

In [23-27], a statement and a method for solving the problem of axisymmetric vibrations of a physically nonlinear viscoelastic cylindrical shell with lumped masses are presented. The function characterizing the deviation of the stress intensity curve from the Hooke's straight line is taken in the form of cubic nonlinearity. A mathematical model, a solution method and a computational algorithm for the problem of axisymmetric vibrations of a cylindrical shell with a concentrated mass, taking into account the physically nonlinear deformation of the material under various boundary conditions, are developed within the framework of the Kirchhoff-Love hypothesis. To solve the resulting system with the Koltunov-Rzhanitsyn weakly singular kernel, a numerical method was applied based on the use of quadrature formulas.

An important stage in the study of the dynamic behavior of the structure under consideration is the determination of the dynamic characteristics of the structure, which include eigenfrequencies and vibration modes, amplitude-phase frequency response, dynamic influence coefficients (dynamic rigidity and dynamic yielding), etc. This is initial information for the subsequent analysis of structural vibrations.

It is known that high-rise buildings are complex system composed of relatively simple structures, mechanically interconnected and interacting in the process of coupled vibrations. This significantly complicates the task of studying its dynamic characteristics by experimental and computational methods. At that, the difficulties that arise can be of both technical and organizational nature. So, for example, a structure may be too large for vibration testing, therefore, the only possibility to calculate complex high-rise structures is to build numerical algorithms and to numerically simulate strain processes.

Thus, the problem of adaptation to the equations of linear viscoelasticity, nonlinear viscoelasticity of the existing apparatus for solving continuous elastic problems contained in the FEM and FDM packages is quite urgent and incomplete, despite a sufficient number of publications on this issue. From this point of view, this work can be interpreted as one of the attempts to implement this concept.

\section{Methods}

Unsteady-state forced vibrations of a high axisymmetric structure are considered; the structure is represented by a one-dimensional model - a viscoelastic beam of annular cross section with a variable slope of the generatrix and a variable thickness. Let us consider an element of the structure shown in Fig. 1 as a design scheme.

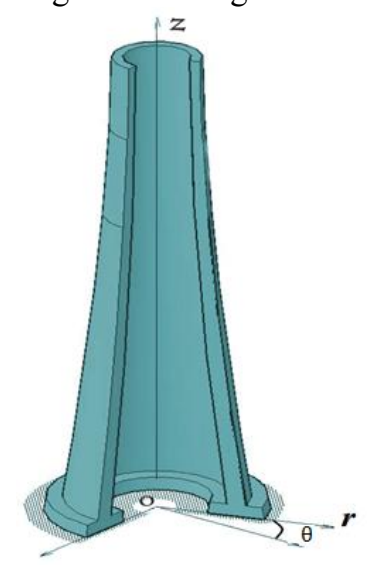

Fig. 1. Design scheme of the structure 
The lower end of the beam $(z=0)$ is rigidly fixed and the kinematic effect $w_{0}(t)$ is set on it; the upper end $(z=L)$ is free. The beam material is a nonlinearly viscoelastic one. Bending unsteady-state forced vibrations of points located at different levels of a structure under set kinematic effect are to be determined.

The mathematical statement of the problem includes the variational equation of the principle of virtual displacements, according to which the sum of work of all active forces, including inertia forces, on a virtual displacement $\delta \mathrm{w}$, satisfying geometrical boundary conditions is zero

$$
\delta A_{M}+\delta A_{u}+\delta A_{P}=0
$$

Here $\delta \mathrm{A}_{\mathrm{M}}, \delta \mathrm{A}_{\text {и }}, \delta \mathrm{A}_{\mathrm{P}}$ - are the virtual work of the bending moment, inertial forces and external forces, respectively, calculated by the formulas:

$$
\delta A_{M}=-\int_{0}^{L} M(z) \delta\left(\frac{\partial^{2} w}{\partial z^{2}}\right) d z, \delta A_{u}=-\rho \int_{0}^{L} F(z)\left(\frac{\partial^{2} w}{\partial^{2}}\right) \delta w d z, \delta A_{P}=\int_{0}^{L} P(z, t) \delta w d z
$$

where $\rho$ - is the beam material density, $L$ - the beam length, $w(z)$ - the beam deflection, $M(z)$ - the bending moment; $F(z)$ - the cross-sectional area; $P(z, t)$ - the external dynamic forces.

Variational problem (1) with dependencies (2) is solved under the following boundary and initial conditions:

$$
z=0: w(z, t)=w_{0}(t), w(z, 0)=u_{0}, \frac{\partial w(z, 0)}{\partial t}=\dot{u}_{0}
$$

where $\mathrm{w}_{0}(\mathrm{t})$ - is the known time function, $u_{0}, \dot{u}_{0}$ are the given constants.

The physical relationship between stresses and strains is taken on the basis of the nonlinear theory of viscoelasticity [8]

$$
\sigma_{z}=E\left\{\left[\varepsilon_{z}(t)-\int_{0}^{t} R_{1}(t-\tau) \varepsilon_{z}(\tau) d \tau\right]-\gamma\left[\varepsilon_{z}^{3}(t)-\int_{0}^{t} R_{2}(t-\tau) \varepsilon_{z}^{3}(\tau) d \tau\right]\right\}
$$

where $\mathrm{E}$ - is the instantaneous modulus of elasticity of the material; $\mathrm{R}_{1}, \mathrm{R}_{2}$ are the relaxation kernels; $\gamma=$ const $>0$ is the non-linearity coefficient, depending on the material of the beam.

In particular, the geometric and physical relations can be taken in the following form

$$
\varepsilon_{z}=-x \frac{\partial^{2} w}{\partial z^{2}}, \quad M_{z}=\int_{F} x \sigma_{z} d F
$$

The problem of unsteady-state nonlinear forced vibrations of a beam consists in the following: for a given function $\mathrm{w}_{0}(\mathrm{t})$ under initial conditions $u_{0}, \dot{u}_{0}$ - to find the deflection $w(z, t)$, strain $\left.\varepsilon_{\mathrm{z}}(\mathrm{z}, \mathrm{t}),\right)$, stress $\sigma_{\mathrm{z}}(\mathrm{z}, \mathrm{t})$ and bending moment $\mathrm{M}_{\mathrm{z}}(\mathrm{z}, \mathrm{t})$, satisfying equations $(1),(2),(4)-(5)$ and conditions (3), (4) for any possible $\delta \mathrm{w}$.

To reduce the above-stated variational problem to a system of resolving equations, the finite element method was used, where a one-dimensional element in the form of a truncated cone working in bending with four degrees of freedom was chosen as a finite element (Fig. 2). 


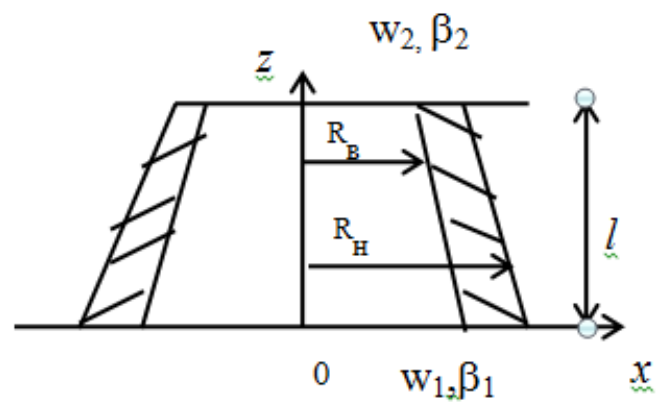

Fig. 2. Used finite element

To reduce the variational problem posed above to a system of resolving equations, the finite element method is used [28], where a one-dimensional element is selected as the finite element, taken in the form of a truncated cone that works on bending with four degrees of freedom [32].

For the displacement function $w$ inside the $e$-th element, the cubic approximation is used:

$$
w=\alpha_{1}+\alpha_{2} z+\alpha_{3} z^{2}+\alpha_{4} z^{3}
$$

then for the first and second derivatives the following expressions are obtained

$$
\frac{\partial w}{\partial z}=\alpha_{2}+2 \alpha_{3} z+3 \alpha_{4} z^{2} ; \frac{\partial^{2} w}{\partial z^{2}}=2 \alpha_{3}+6 \alpha_{4} z
$$

The dependence of the nodal displacements and rotation angles of the $e$-th finite element $\left\{\mathrm{w}_{\mathrm{i}}\right\}$ on the vector of arbitrary constants $\left\{\alpha_{\mathrm{i}}\right\}$ in matrix form is written as

$$
\left\{w_{i}\right\}=\left[\begin{array}{cccc}
1 & 0 & 0 & 0 \\
0 & 1 & 0 & 0 \\
1 & l & l^{2} & l^{3} \\
0 & 1 & 2 l & 3 l^{2}
\end{array}\right]\left\{\alpha_{i}\right\}
$$

where $\left\{w_{i}\right\}^{T}=\left\{w_{i}, \beta_{1}, w_{2}, \beta_{2}\right\}, \beta_{1}=\left.\frac{\partial w}{\partial z}\right|_{z=0}, \beta_{2}=\left.\frac{\partial w}{\partial z}\right|_{z=l}$.

Hereinafter, the following notation is used: \{\}$-$ vector, [] - matrix, ${ }^{\mathrm{T}}$ - transposition operation.

The transformation inverse to (8), i.e. the matrix dependence of $\left\{\alpha_{i}\right\}$ on $\left\{\mathrm{w}_{\mathrm{i}}\right\}$ is expressed as

$$
\left\{\alpha_{i}\right\}=\left[\begin{array}{cccc}
1 & 0 & 0 & 0 \\
0 & 1 & 0 & 0 \\
-\frac{3}{l^{2}} & -\frac{2}{l} & \frac{3}{l^{2}} & -\frac{1}{l} \\
\frac{2}{l^{3}} & \frac{1}{l^{2}} & -\frac{2}{l^{3}} & \frac{1}{l^{2}}
\end{array}\right]\left\{w_{i}\right\} \text {, i.e. }\left\{\alpha_{i}\right\}=[A]\left\{w_{i}\right\}
$$

Using the indicated transformations (11), we express the displacement function (8) and its derivatives in a matrix form in terms of nodal displacements $\left\{\mathrm{w}_{\mathrm{i}}\right\}$ 
We introduce the matrix [B]

$$
\begin{gathered}
\left.w=\left\lfloor 1 \cdot z \cdot \cdot z^{2} \cdot \cdot z^{3}\right\rfloor\left\{\alpha_{i}\right\}=\left\lfloor 1 \cdot z^{\prime} \cdot z^{2} \cdot z^{3}\right\rfloor A\right]\left\{w_{i}\right\} \\
\left.\frac{\partial w}{\partial z}=\left[0 \cdot 1 \cdot \cdot 2 z \cdot 3 z^{2}\right] A\right]\left\{w_{i}\right\} \\
\frac{\partial^{2} w}{\partial z^{2}}=[0 \cdot 0 \cdot \cdot 2 \cdot 6 z][A]\left\{w_{i}\right\}=
\end{gathered}
$$

then

$$
[B]=[0 \cdot 0 \cdot \cdot 2 \cdot 6 z][A]\left[-\frac{6}{l^{2}}+\frac{12 z}{l^{3}} ;-\frac{4}{l}+\frac{6 z}{l^{2}} ; \frac{6}{l^{2}}-\frac{12 z}{l^{3}} ;-\frac{2}{l}+\frac{6 z}{l^{2}}\right],
$$

$$
\frac{\partial^{2} w}{\partial z^{2}}=[B]\left\{w_{i}\right\}
$$

Substituting expression (3-5) in (2), we obtain the virtual work of the bending moment for the $e$-th element

$$
\begin{aligned}
& \delta A_{M}^{e}=\int_{0}^{l}\left[\frac{\partial^{2} w}{\partial z^{2}} \delta\left(\frac{\partial^{2} w}{\partial z^{2}}\right) \int_{F} x^{2} d F\right] d z-E \int_{0}^{t}\left\{R_{1}(t-\tau) \int_{0}^{l}\left[\frac{\partial^{2} w}{\partial z^{2}} \delta\left(\frac{\partial^{2} w}{\partial z}\right) \int_{F} x^{2} d F\right] d z\right\} d \tau \\
& -E \gamma \int_{0}^{l}\left[\left(\frac{\partial^{2} w}{\partial z^{2}}\right)^{3} \delta\left(\frac{\partial^{2} w}{\partial z^{2}}\right) \int_{F} x^{4} d F\right] d z+E \gamma \int_{0}^{t}\left\{R_{2}(t-\tau) \int_{0}^{l}\left[\left(\frac{\partial^{2} w}{\partial z^{2}}\right)^{3} \delta\left(\frac{\partial^{2} w}{\partial z}\right) \int_{F} x^{4} d F\right] d z\right\} d \tau
\end{aligned}
$$

Substitution of (13) into (14) and integration over the cross-sectional area leads each term of expression (14) to the following form:

the first term

$$
\delta\left\{w_{i}\right\}^{T} E \int_{0}^{l} J^{e}(z)[B]^{T}[B] d z\left\{w_{i}\right\}=\delta\left\{w_{i}\right\}^{T}\left[K^{e}\right]\left\{w_{i}\right\}
$$

where

$$
J^{e}(z)=\frac{\pi}{4}\left[\left(R_{H}(z)\right)^{4}-\left(R_{B}(z)\right)^{4}\right] \text { - is the moment of inertia of the cross section of the }
$$

$e$-th element; $R_{H}, R_{B}$ are the outer and inner radii of the element, respectively;

$$
\left[K^{e}\right]=E \int_{0}^{l} J^{e}(z)[B]^{T}[B] d z \text { - is the stiffness matrix of the } e \text {-th element. }
$$

the second term

$$
E \int_{0}^{t}\left\{R_{1}(t-\tau) \int_{0}^{l}\left[\frac{\partial^{2} w}{\partial z^{2}} \delta\left(\frac{\partial^{2} w}{\partial z^{2}}\right) \int_{F} x^{2} d F\right] d z\right\} d \tau=\delta\left\{w_{i}\right\}^{T} \int_{0}^{t}\left\{R_{1}(t-\tau)\left[K^{e}\right]\left\{w_{i}\right\} d \tau\right.
$$

the third term

$$
E \gamma \int_{0}^{l}\left[\left(\frac{\partial^{2} w}{\partial z^{2}}\right)^{3} \delta\left(\frac{\partial^{2} w}{\partial z^{2}}\right) \int_{F} x^{4} d F\right] d z=\delta\left\{w_{i}\right\}^{T} E \gamma \int_{0}^{l} J_{1}^{e}(z)[B]^{T}[B]\left\{w_{i}\right\}[B]\left\{w_{i}\right\}[B]\left\{w_{i}\right\} d z
$$


Here $J^{e}(z)=\frac{\pi}{8}\left[\left(R_{H}(z)\right)^{6}-\left(R_{B}(z)\right)^{6}\right]$.

Expanding the expression under the integral sign in (17):

$$
\begin{aligned}
& \left\{V^{e}\right\}=J_{1}^{e}(z)\left[-\frac{6}{l^{2}}+\frac{12 z}{l^{3}} ;-\frac{4}{l}+\frac{6 z}{l^{2}} ; \frac{6}{l^{2}}-\frac{12 z}{l^{3}} ;-\frac{2}{l}+\frac{6 z}{l^{2}}\right]^{T}\left[\left(-\frac{6}{l^{2}}+\frac{12 z}{l^{3}}\right) w_{1}+\right. \\
& \left.\left(-\frac{4}{l}+\frac{6 z}{l^{2}}\right) \varphi_{1}+\left(\frac{6}{l^{2}}-\frac{12 z}{l^{3}}\right) w_{2}+\left(-\frac{2}{l}+\frac{6 z}{l^{2}}\right) \varphi_{2}\right]^{3}
\end{aligned}
$$

we see that it is a vector whose coordinates are cubic polynomials from nodal displacements.

As a result of integration over the length of the element, the third term (17) is

$$
E \gamma \int_{0}^{l}\left[\left(\frac{\partial^{2} w}{\partial z^{2}}\right)^{3} \delta\left(\frac{\partial^{2} w}{\partial z^{2}}\right) \int_{F} x^{4} d F\right] d z=\delta\left\{w_{i}\right\}^{T} E \gamma\left\{V^{e}\right\}
$$

where the index "e" indicates that the vector $\{\mathrm{Ve}\}$ is defined for the $e$-th element.

The fourth term

$$
\begin{aligned}
& E \gamma \int_{0}^{t}\left\{R_{2}(t-\tau) \int_{0}^{l}\left[\left(\frac{\partial^{2} w}{\partial z^{2}}\right)^{3} \delta\left(\frac{\partial^{2} w}{\partial z}\right) \int_{F} x^{4} d F\right] d z\right\} d \tau= \\
= & \delta\left\{w_{i}\right\}^{T} E \gamma \int_{0}^{t} R_{2}(t-\tau) \int_{0}^{l} J_{1}^{e}(z)[B]^{T}[B]\left\{w_{i}\right\}[B]\left\{w_{i}\right\} d z d \tau
\end{aligned}
$$

Considering (20), we get

$$
E \gamma \int_{F} x^{5} \int_{0}^{l} R_{2}(t-\tau)\left(\frac{\partial^{2} w}{\partial z^{2}}\right)^{3} \delta\left(\frac{\partial^{2} w}{\partial z^{2}}\right) d z d F=\delta\left\{w_{i}\right\}^{T} E \gamma \int_{0}^{t} R_{2}(t-\tau) d \tau\left\{V^{e}\right\}
$$

The use of the finite element method procedure leads the variational problem (1) and (3) to a nonlinear system of integro-differential equations, which has the following matrix form:

$$
\begin{aligned}
& {[M]\{\ddot{w}(t)\}+[K]\{w(t)\}=\{P(t)\}-\int_{0}^{t} R_{1}(t-\tau)[K]\{w(t) d \tau+E \gamma\{V(t)\}\}-} \\
& E \gamma \int_{0}^{t} R_{2}(t-\tau) d \tau\{V(t)\}
\end{aligned}
$$

Here $[\mathrm{M}],[\mathrm{K}]$ are the matrices of mass and rigidity of the entire structure; $\{\mathrm{w}\}$ is the displacement vector of all the nodal points of the structure; $\{\mathrm{V}\}$ is a vector whose coordinates are determined by cubic polynomials of system displacements, $\{\mathrm{P}\}$ is a vector of external influences.

This equation is solved by the Newmark method [29-30]. Equation (22) at given initial conditions (4) is solved by direct integration using a numerical step-by-step procedure. We used the Newmark method to solve the system of equations (22), based on independent expansions of $w\left(t_{i}+\tau\right)$ and its derivative into the series in powers $\tau$, while holding the terms containing the third derivative $w_{i}$. The coefficients for the residual terms $\alpha$ and $\beta$ are selected from the condition for ensuring the unconditional convergence of the integration process: 


$$
\begin{gathered}
\mathrm{w}\left(\mathrm{t}_{\mathrm{i}}+\tau\right)=\mathrm{w}_{\mathrm{i}}+\tau \dot{\mathrm{w}}_{\mathrm{i}}+\frac{\tau^{2}}{2} \ddot{\mathrm{w}}_{\mathrm{i}}+\alpha \tau^{3} \dddot{\mathrm{w}}_{\mathrm{i}} \\
\dot{\mathrm{w}}\left(\mathrm{t}_{\mathrm{i}}+\tau\right)=\dot{\mathrm{w}}_{\mathrm{i}}+\tau \ddot{\mathrm{w}}_{\mathrm{i}}+\beta \tau^{2} \dddot{\mathrm{w}}_{\mathrm{i}}
\end{gathered}
$$

Substituting $\dddot{\mathrm{W}}_{\mathrm{i}}=\frac{\ddot{\mathrm{W}}_{\mathrm{i}+1}-\ddot{\mathrm{W}}_{\mathrm{i}}}{\tau}$, expressions for displacements and velocities (23) are written as

$$
\begin{gathered}
\mathrm{w}_{\mathrm{i}+1}=\mathrm{w}_{\mathrm{i}}+\tau \dot{\mathrm{W}}_{\mathrm{i}}+\frac{\tau^{2}}{2} \ddot{\mathrm{w}}_{\mathrm{i}}+\alpha \tau^{3}\left(\ddot{\mathrm{w}}_{\mathrm{i}+1}-\ddot{\mathrm{w}}_{\mathrm{i}}\right) \\
\dot{\mathrm{w}}_{\mathrm{i}+1}=\mathrm{w}_{\mathrm{i}}+\tau \ddot{\mathrm{w}}_{\mathrm{i}}+\beta \tau^{2}\left(\ddot{\mathrm{w}}_{\mathrm{i}+1}-\ddot{\mathrm{w}}_{\mathrm{i}}\right)
\end{gathered}
$$

Then the acceleration obtained form (26)

$$
\ddot{\mathrm{w}}_{\mathrm{i}+1}=\frac{1}{\alpha \tau^{2}}\left(\mathrm{w}_{\mathrm{i}+1}-\mathrm{w}_{\mathrm{i}}\right)-\frac{1}{\alpha \tau} \dot{\mathrm{w}}_{\mathrm{i}}+\left(1-\frac{1}{2 \alpha}\right) \ddot{\mathrm{w}}_{\mathrm{i}}
$$

is substituted into the velocity expression (27)

$$
\dot{\mathrm{w}}_{\mathrm{i}+1}=\frac{\beta}{\alpha \tau}\left(\mathrm{w}_{\mathrm{i}+1}-\mathrm{w}_{\mathrm{i}}\right)+\left(1-\frac{\beta}{\alpha}\right) \mathrm{w}_{\mathrm{i}}+\frac{\tau}{2}\left(2-\frac{\beta}{\alpha}\right) \ddot{\mathrm{w}}_{\mathrm{i}}
$$

To find a solution $w_{i+1}$ for time $\mathrm{t}_{\mathrm{i}+1}$, the general equation of motion is written as follows:

$$
[M] \ddot{w}_{i+1}+[C] \dot{w}_{i+1}+[K] w_{i+1}=\left\{P_{i+1}\right\}
$$

After substituting expressions for accelerations (28) and velocity (29) into (30) an algebraic system of equations is obtained

$$
[A]\left\{w_{i+1}\right\}=\left\{R_{i+1}\right\}
$$

Where

$$
\begin{gathered}
{[A]=[K]+\frac{1}{\alpha \tau^{2}}[M]} \\
\left\{R_{i+1}\right\}=\left\{P_{i+1}\right\}+[M]\left(\frac{1}{\alpha \tau^{2}}\left\{w_{i}\right\}+\frac{1}{\alpha \tau}\left\{\dot{w}_{i}\right\}+\left(\frac{1}{2 \alpha}-1\right)\left\{\ddot{w}_{i}\right\}\right)+\left\{W_{i}\right\}
\end{gathered}
$$

where

$$
\left\{W_{i}\right\}=\int_{0}^{t} R_{1}(t-\tau)[K]\left\{w_{i}\right\} d \tau+E \gamma\left\{V_{i}\right\}-E \gamma \int_{0}^{t} R_{2}(t-\tau) d \tau\left\{V_{i}\right\}
$$

To solve the resulting system of equations (31), it is necessary to specify at the initial moment the values of displacements $\left\{w_{0}\right\}$, velocity $\left\{\dot{\mathrm{w}}_{0}\right\}$ and accelerations $\left\{\ddot{\mathrm{W}}_{0}\right\}$. Usually $\left\{\ddot{w}_{0}\right\}=0$ is taken. The Newmark method is unconditionally stable if

$$
\beta \geq 0.5, \alpha \geq 0,25(\beta+0,5)^{2},
$$

\section{Results and Discussion}

Thus, the algorithm that implements the Newmark method for solving the matrix system of nonlinear differential equations (22) obtained in the course of finite element discretization with the initial condition (4) is as follows: 
1. The initial values are set $\left\{\mathrm{w}_{0}\right\},\left\{\dot{\mathrm{w}}_{0}\right\}$.

2. A system of algebraic equations (29) is formed, the right-hand side of which contains nonlinear terms that determine the viscoelastic and nonlinear-viscous properties of the material, depending on the deformed state reached by the system.

When accounting for the viscoelastic properties of the material with the above formulation, terms containing cubic terms from displacements are excluded in the right-hand side of the resulting resolving algebraic system of equations (22). In this case, the equation takes the following form

$$
[\mathrm{M}]\{\ddot{\mathrm{W}}(\mathrm{t})\}+[\mathrm{K}]\{\mathrm{w}(\mathrm{t})\}=\{\mathrm{P}(\mathrm{t})\}+\int_{0}^{t} R_{1}(t-\tau)[K]\{\mathrm{w}(\mathrm{t})\} \mathrm{d} \tau
$$

with homogeneous initial conditions:

$$
w(z, 0)=0, \frac{\partial w(z, 0)}{\partial t}=0
$$

The task is to determine the displacements of the points of the structure at different time points. The resulting nonlinear system of integro-differential equations (33) with initial conditions (34) is solved by the Newmark method.

The algorithm that implements the Newmark method for solving the matrix system of nonlinear integro-differential equations obtained in the course of finite element discretization (33) is as follows:

1. The initial values are set $\left\{\mathrm{w}_{0}\right\},\left\{\dot{\mathrm{w}}_{0}\right\}$.

2. A system of algebraic equations (29) with a linear right-hand side is formed, i.e. for $\left\{\mathrm{W}_{\mathrm{i}}(\mathrm{t})\right\}=0$.

3. The system of linear algebraic equations (29) is solved, as a result of which the current value of the displacement vector $\left\{\mathrm{w}_{\mathrm{i}+1}\right\}$ is determined.

4. The formulas of Newmark's method (26), (27) are used to determine the vectors of velocity and acceleration at the current time point $t_{i+1}$.

5. The value of integral $\int_{t_{i-1}}^{t_{i}} R_{1}\left(t_{i}-\tau\right) \mathrm{d} \tau$ on the segment $\left(\mathrm{t}_{\mathrm{i}-1}, \mathrm{t}_{\mathrm{i}}\right)$ is calculated by the formulas of numerical integration, for example, by the approximate formula of averages or by the formula of trapezoids. In the first case, the approximate value of the integral is defined as the product of the integrand at the point $t_{i}-\tau / 2$ by the length of the segment $\tau$. For $\mathrm{i}=1 \mathrm{t}_{\mathrm{i}-\mathrm{1}}=0$. In the second case, the value of the integral is approximately equal to

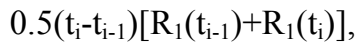

6 . The resulting value of the integral is multiplied by the vector $[\mathrm{K}]\left\{\mathrm{w}_{\mathrm{i}}\right\}$ and this vector is added to the right-hand side of the system (29).

7. Steps 3 - 6 are repeated until the end of the process.

In this formulation, with the developed methodology and the created computer program, a number of problems, previously investigated in a linearly elastic formulation, were solved [31-33]. In all the examples considered below, the values of the viscosity parameters are taken as $\mathrm{R}_{1} \mathrm{~A}=0.0194 ; \beta=0.00000014 ; \alpha=0.075, \mathrm{R}_{2}=2 \mathrm{R}_{1} ; \gamma=120000$ [34].

As seen from the comparison (Fig. 3) of the presented results of forced vibrations of the pipe at the initial period, the elastic and viscoelastic solutions practically do not differ. Then, over time, the vibrations of a viscous pipe begin to differ markedly from the vibrations of an elastic pipe, the amplitude of which increases linearly. The amplitude of vibrations of points of a pipe with viscoelastic characteristics of the material, after reaching a certain maximum value, begins to gradually decrease. 
Fig. 4 shows the results of forced vibrations of the pipe under sinusoidal damping effect.

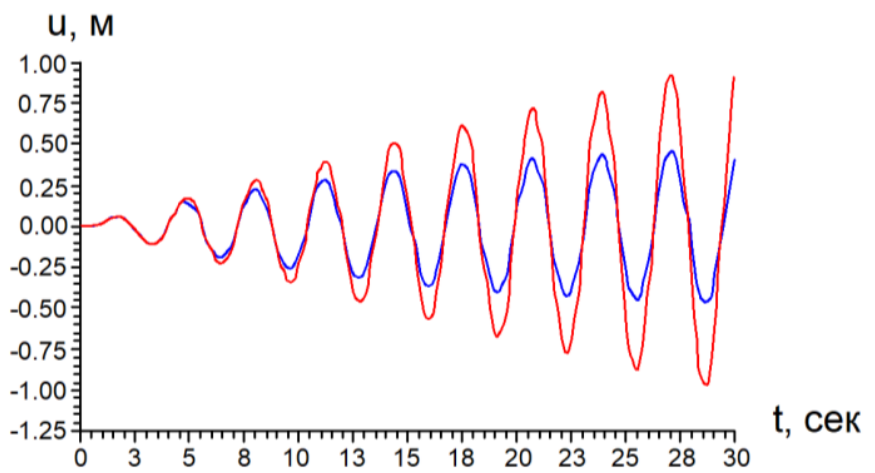

Fig. 3. Forced vibrations of the point $\mathrm{z}=325 \mathrm{~m}$ of the pipe at resonance mode $\ddot{\mathrm{W}}_{0}=0.1 \mathrm{~A} \sin (1.68 \mathrm{t})$ : - viscoelastic solution; -elastic solution

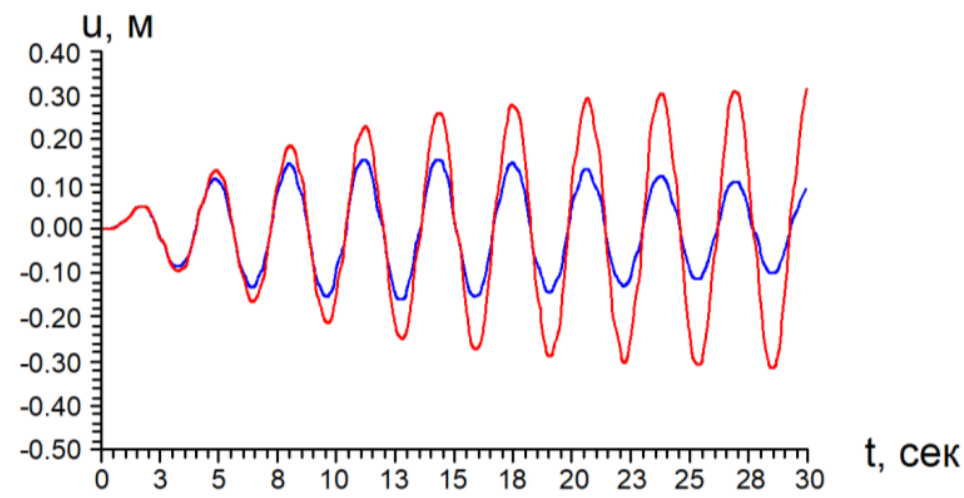

Fig. 4. Forced vibrations of a point $(\mathrm{z}=325 \mathrm{~m})$ of a pipe under the impact $\ddot{\mathrm{W}}_{0}$ $=0.1 \mathrm{~A} \sin (1.68 \mathrm{t}) \exp (-0.1 \mathrm{t})$ : - viscoelastic solution; -elastic solution

Let us consider the forced unsteady vibrations of a high-rise pipe taking into account the viscoelastic properties of the material when the horizontal component of the real accelerogram of the Gazli earthquake acts on the base of the structure [35].

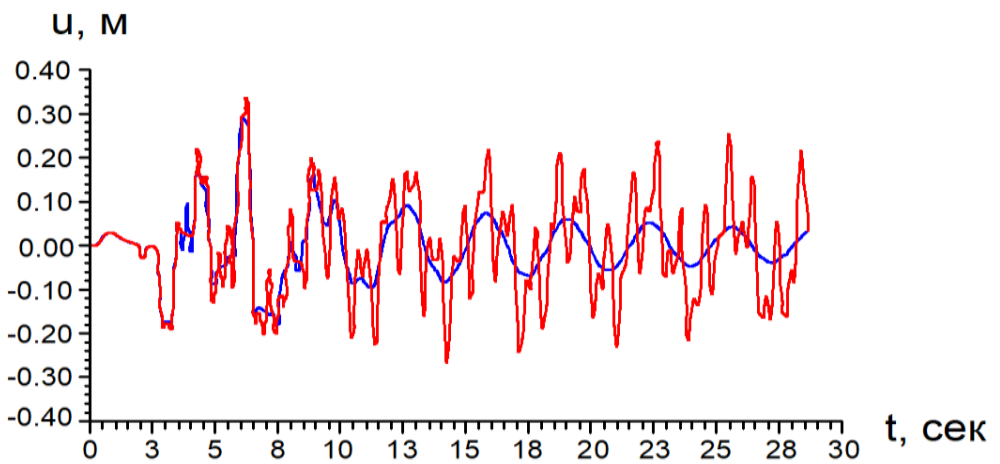

Fig. 5 Forced vibrations of the point $(z=325 \mathrm{~m})$ of the pipe under the effect of the accelerogram of the Gazli earthquake: -elastic solution 
The results of solving the problem (Fig. 5) show that, at the initial period, the behavior of a viscoelastic structure does not differ from the behavior of an elastic one. Subsequently, an account for the viscoelastic properties of the structure material leads to a noticeable decrease in the vibration amplitude, high frequencies damp and the vibrations of the viscoelastic structure have a pattern of free damped vibrations with the fundamental frequency of natural vibrations. It is seen here that an account for the viscoelastic properties of the material, somewhat averages the displacements of the points of the pipe, leaving as significant only the oscillations of the fundamental mode.
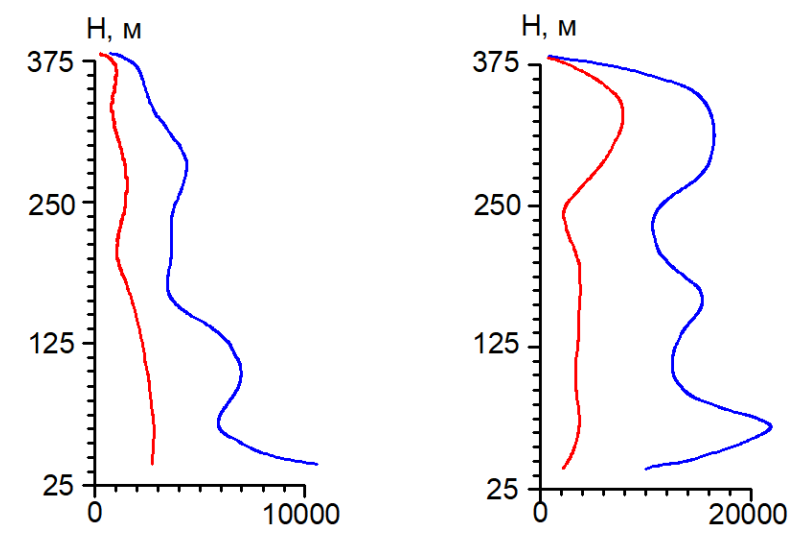

Fig. 6. Diagrams of the maximum values of shearing forces (a) and bending moments (b) arising in the pipe under the impact of a real accelerogram: solution

Fig. 6 shows a comparison of the diagrams of the maximum values of shear forces and bending moment obtained under unsteady forced vibrations resulting from the impact of the high-frequency accelerogram of the Gazli earthquake on structures. The line with asterisks is the result of the elastic solution, and the solid line is the result of the viscoelastic solution. As seen from Fig. 6, higher eigenfrequencies are also manifested in an elastic structure under high-frequency effect; therefore, among the modes of vibrations of the structure, there are modes corresponding to higher frequencies. An account for the viscoelastic properties of the material not only significantly reduces the force factors arising in the body of the structure, and distributed in proportion to the mode of vibration achieved under a given impact, but also smoothers them.

It should be noted that if the viscoelastic properties of the material are not taken into account, then the calculation gives the maximum values of the shear forces at the base of a high-rise structure while considering the viscoelastic properties of the material, the maximum values are reached at a certain height from the base. In addition, significant (though not maximum) forces arise in the upper part, at the level of $\sim 2 / 3 \mathrm{H}$ ( $\mathrm{H}$ is the height of the structure).

During a survey of the consequences of the earthquake in Fukui (Japan) on June 28, 1948, the destruction of more than 40 reinforced concrete smokestacks were observed at the level of $2 / 3$ of the height. The destruction of pipes at this very point was explained by the lack of the methods used for calculating such unique structures and not considering a number of factors that describe the real properties of the material. In relatively flexible structures, during an earthquake, great forces can occur due to the higher modes of natural vibrations the developed technique takes this into account.

Now let us consider a general case when both nonlinear and viscoelastic properties of the material are taken into account. In this case, the above formulation does not allow any 
simplifications on the right-hand side of the resolving system of nonlinear integrodifferential equations (22). The equation has the form

$$
\begin{aligned}
{[\mathrm{M}]\{\ddot{\mathrm{W}}(\mathrm{t})\}+[\mathrm{K}]\{\mathrm{w}(\mathrm{t})\}=} & \{\mathrm{P}(\mathrm{t})\}+\int_{0}^{t} R_{1}(t-\tau)[K]\{\mathrm{w}\} \mathrm{d} \tau+\mathrm{EJ}_{1} \gamma\{\mathrm{V}(\mathrm{t})\}- \\
& -\mathrm{EJ}_{1} \gamma \int_{0}^{t} R_{2}(t-\tau) d \tau\{\mathrm{V}(\mathrm{t})\}
\end{aligned}
$$

with homogeneous initial conditions (34).

The task is to determine the displacements of the points of the structure at different time points. The nonlinear system of integro-differential equations (22) with initial conditions (34) is solved by the Newmark method.

The algorithm that implements the Newmark method for solving the matrix system of nonlinear integro-differential equations obtained in the course of finite element discretization (22) is as follows:

1. The initial values are set $\left\{\mathrm{w}_{0}\right\},\left\{\dot{\mathrm{W}}_{0}\right\}$.

2. A system of algebraic equations (31) with a linear right-hand side is formed, i.e. for $\left\{\mathrm{W}_{\mathrm{i}}\right\}=0$.

3. The system of linear algebraic equations (29) is solved, as a result of which the current value of the displacement vector $\left\{\mathrm{w}_{\mathrm{i}+1}\right\}$ is determined.

4. The formulas of Newmark's method (26), (27) are used to determine the vectors of velocity and acceleration at the current time moment $t_{i+1}$.

5. The coordinates of the nonlinear vector $\left\{\mathrm{W}_{\mathrm{i}}\right\}=\mathrm{E} \gamma\left\{\mathrm{V}_{\mathrm{i}}\right\}$, which are cubic polynomials in the found nodal displacements and rotation angles are calculated using formulas (31).

6. The resulting vector is added to the right-hand side of the system (29).

7. By the formulas of numerical integration, for example, by the approximate formula of averages, the value of the integral $\int_{t_{i-1}}^{t_{i}} R_{1}\left(t_{i}-\tau\right) \mathrm{d} \tau$ on the segment $\left(\mathrm{t}_{\mathrm{i}-1}, \mathrm{t}_{\mathrm{i}}\right)$ is calculated as the product of the integrand at point $t_{i}-\tau / 2$ by the length of the segment $\tau$. For $i=1 t_{i-1}=0$.

8. The resulting value of the integral is multiplied by the vector $[\mathrm{K}]\left\{\mathrm{w}_{\mathrm{i}}\right\}$ and this vector is added to the right-hand side of the system (31).

9. The product of the nonlinear vector $\left\{\mathrm{W}_{i}\right\}$ by the integral $\int_{t_{i-1}}^{t_{i}} R_{2}\left(t_{i}-\tau\right) d \tau$ is found.

This product is also added to the right-hand side of the system (29).

10. Steps 3 - 9 are repeated until the end of the process.

In such a general statement, with the developed methodology and the created computer program, problems are solved in linear, nonlinear, and viscoelastic formulations [32-33]. In all the examples considered below, the values of the viscosity parameters are taken as $R_{1}$ $\mathrm{A}=0,0194 ; \beta=0,00000014 ; \alpha=0,075, \mathrm{R}_{2}=2 \mathrm{R}_{1} ; \gamma=120000$.

Let us consider the forced vibrations of a nonlinear-visco-elastic high-rise pipe under kinematic excitation of the base according to the harmonic law [32]. The obtained horizontal displacements for the pipe point $z=325 \mathrm{~m}$ are shown in Fig. 7. A line with asterisks corresponds to these displacements. Here, for comparison, the solid line represents the solution for the same point of a linearly elastic structure. 


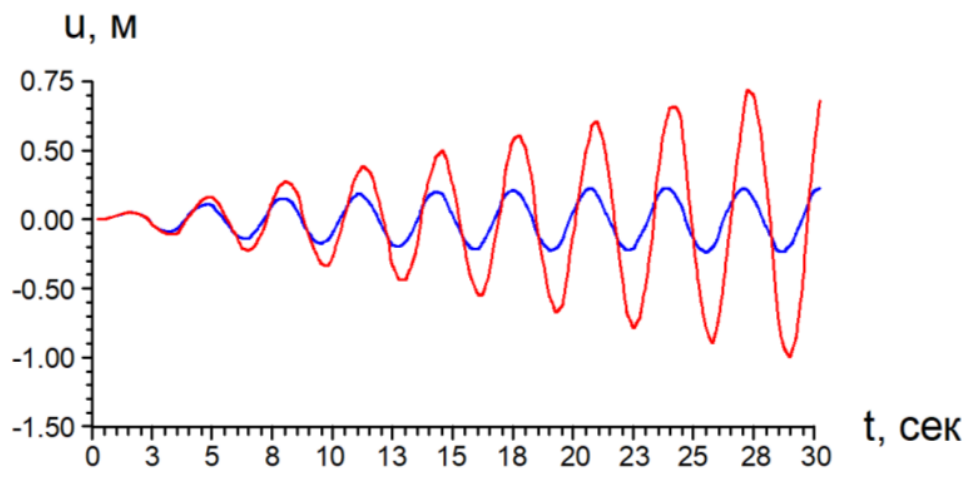

Fig. 7 Forced vibrations of the point $\mathrm{z}=325 \mathrm{~m}$ of the pipe taking into account the nonlinear viscoelastic properties of the material under the impact $\ddot{\mathrm{W}}_{0}=0.1 \mathrm{~A} \sin (1.68 \mathrm{t})$ : — - nonlinearviscoelastic solution; - linear-elastic solution

The analysis of the results presented shows that the general case, when nonlinear and viscous properties of the material are taken into account, leads to the greatest decrease in the amplitudes of displacements of the points of a high-rise structure in comparison with all previous options. Fig. 8 shows the results for sinusoidal damping of kinematic impact [33] at the base of the pipe.

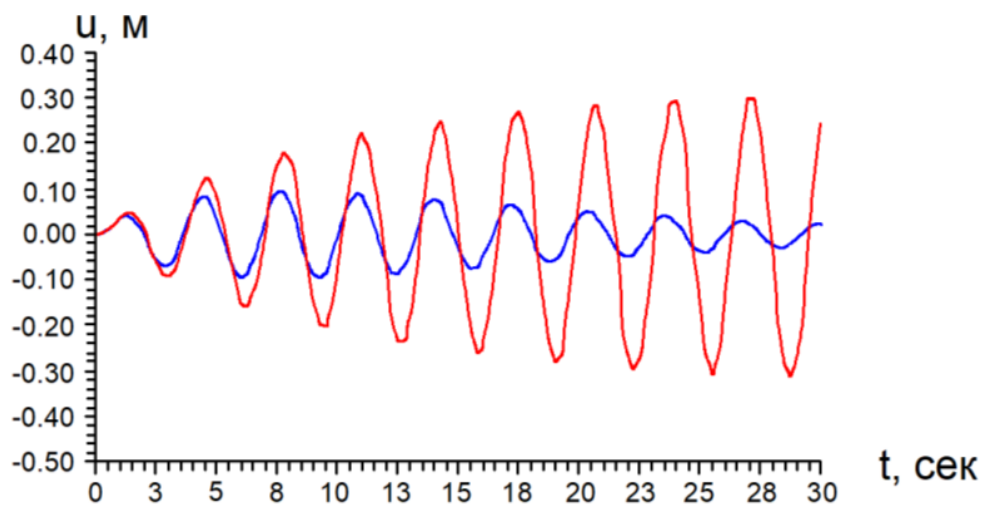

Fig. 8. Forced vibrations of the point $\mathrm{z}=325 \mathrm{~m}$ of the pipe, taking into account the nonlinear viscoelastic properties of the material under the impact $\ddot{\mathrm{W}}_{0}=0.1 \mathrm{~A} \sin (1.68 \mathrm{t}) \exp (-0.1 \mathrm{t})$ : — - nonlinear-viscoelastic solution; - linear-elastic solution

Thus, the study of the dynamic behavior of a high-rise structure taking into account the nonlinear and dissipative properties (different in nature) of the material, shows that the joint consideration of all these properties brings the resulting pattern closer to the one observed in reality. That is, the oscillation amplitude of the structure does not grow infinitely, but gradually decreases over time, and the maximum possible consideration of nonlinear and dissipative properties leads to the fastest damping of oscillations.

\section{Conclusions}

Based on the above studies, the following conclusions can be drawn: 
1. A methodology and an algorithm for studying forced vibrations of high-rise structures in linear, nonlinear, and viscoelastic formulations under various kinematic impacts were developed, taking into account the viscoelastic properties of the material.

2. The results of investigations of forced vibrations of a high-rise pipe, considering the viscoelastic properties of the material when the horizontal component of the real accelerogram of the Gazli earthquake acts on the base of the structure, show that an account for the viscoelastic properties of the structure material leads to a noticeable decrease in the amplitude of vibrations. At the same time, high frequencies and vibrations of the viscoelastic structure damp. It can be seen here that an account for the viscoelastic properties of the material somewhat averages the displacements of the pipe points, leaving as significant only the fundamental modes of vibrations.

3. Higher frequencies are also observed in an elastic structure under high-frequency impact, therefore, among the modes of vibrations of the structure, there are modes that correspond to higher frequencies. An account for the viscoelastic properties of the material not only significantly reduces the force factors arising in the body of the structure, which are distributed in proportion to the mode of vibration achieved under a given impact, but also smoothers them.

4. Combined consideration of the nonlinear and dissipative properties of the material brings the resulting pattern closer to the one observed in reality. Here, the vibration amplitude of the structure does not grow indefinitely, but gradually decreases over time. In a dynamic study, the maximum possible consideration of the nonlinear and dissipative properties of the material of structures leads to the fastest damping of the vibration amplitude.

\section{References}

1. M.A. Koltunov, M. Mirsaidov, I.E. Troyanovsky, Polymer Mechanics 14(2), 233-238 (1978)

2. M. Mirsaidov, I.E. Troyanovsky, Polymer Mechanics 11(6), 953-955 (1975)

3. V.I. Myachenkov, E.K. Pavlov, Soviet Applied Mechanics 18(5) 434-441 (1982)

4. V.I. Myachenkov, V. Maltsev, Mechanical Engineering 278-286 (1984)

5. A.A. Maltsev, V.P. Maltsev, V.I. Myachenkov, Mechanics of deformable systems 150-158 (1979)

6. M.A. Koltunov, A.I. Karimov, T. Mavlyanov, Mechanics of Composite Materials 16(5), 591-595 (1981)

7. A.A. Il'yushin, B.E. Pobedrya, Fundamentals of the mathematical theory of thermoviscoelasticity, Science, Moscow (1970)

8. A.A. Il'yushin, P.M. Ogibalov, Polymer Mechanics 2, 111-121 (1966)

9. D. Younesian, A. Hosseinkhani, H. Askari, Nonlinear Dyn 97, 853-895 (2019)

10. A. Muliana, K.R. Rajagopal, A.S. Wineman, Acta Mech 224, 2169-2183 (2013)

11. G.G. Li, Z.Y. Zhu, C.J. Cheng, Mech Time-Depend Mater 7, 175-188 (2003)

12. H.H. Hilton, Mech Time-Depend Mater 13, 1-10 (2009)

13. A.N. Ishmatov, M. Mirsaidov, Soviet Applied Mechanics 27(4), 388-394 (1991)

14. Sh. Khudainazarov, T. Sabirjanov, A. Ishmatov, Journal of Physics: Conf. Series 1425, 012009 (2020)

15. P. Mata, A.H. Barbat, S. Oller, Arch Computat Methods Eng 15, 489 (2008)

16. S.A. Salawu, G.M. Sobamowo, O.M. Sadiq, Iran J Sci Technol Trans Civ Eng 44, 277-288 (2020)

17. A.A. Kaminskii, I.Y. Podil'chuk, Int Appl Mech 34, 1248-1256 (1998)

18. J.J. Li, C.J. Cheng, Nonlinear Dyn 61, 57-70 (2010)

19. W. Song, S. Liang, J. Song, L. Zou, G. Hu, Engineering Structures 195, 414-424 (2019)

20. S.R. Munshi, V.J. Modi, T Yokomizo, Journal of Fluids and Structures 11, 873-892 
(1997)

21. F. Hou, P.P. Sarkar, Engineering Structures 207, 110259 (2020)

22. X. Chen, Journal of Structural Engineering 134, 782-791 (2008)

23. D.A. Khodzhaev, R.A. Abdikarimov, M.M. Mirsaidov, Magazine of Civil Engineering 91(7), 39-48 (2019)

24. M. Mirsaidov, I.I. Safarov, M.K. Teshaev, E3S Web of Conferences 164(5), 140132019 (2020)

25. M.M. Mirsaidov, I.I. Safarov, M.K. Teshaev, Z.I. Boltayev, Journal of Physics: Conference Series 1706(1), 0120331 (2020)

26. T. Mavlanov, Sh. Khudainazarov, I. Khazratkulov, Journal of Physics: Conf. Series 1425, 0120172020

27. T. Mavlanov, Sh. Khudainazarov, E3S Web of Conferences 97, 040542 (2019)

28. J.R.T. Hughes, Dover Publikation, Mineola, New York 675 (2012)

29. N.M. Newmark, Seism response anal of nuclear power plant system, San Francisco USA (1977)

30. R.L. Sharpe, N.M. Newmark, Proceedings of the Annual Offshore Technology Conference, Houston, United States 140861, 177-184 (1977)

31. M.M. Mirsaidov, Sh.O. Khudainazarov, Magazine of Civil Engineering 96(4), 118$128(2020)$

32. Sh.O. Khudainazarov, B. Donayev, J.A. Yarashov, IOP Conf. Series: Materials Science and Engineering 883, 012195 (2020)

33. Sh.O. Khudainazarov, T. Mavlanov, J. Qosimov, O.S. Nurova, IOP Conf. Series: Materials Science and Engineering 869, 1-13 (2020)

34. S.V. Alexandrovsky, Creep and shrinkage of concrete reinforced concrete structures, Streoyizdat, Moscow (1976)

35. V.V. Steinberg, K.T. Pletnev, V.I. Grazer, Accelerogram of oscillations during a devastating earthquake, Earthquake-resistant construction 45-61 (1977) 